\title{
Implante percutáneo de válvula pulmonar: a propósito de dos casos colombianos
}

\author{
Percutaneous pulmonary valve implant: Two Colombian case reports \\ Luis H. Díaz, Rafael Lince, Keerby Hernández*, Rafael Correa y Arnaldo Palomino \\ Servicio de Cardiología Pediátrica, Clínica CardioVID. Medellín, Colombia
}

\section{Resumen}

Los pacientes con cardiopatías congénitas que afectan la continuidad del ventrículo derecho con la arteria pulmonar deben someterse con frecuencia a intervenciones debido a la limitada vida útil de los conductos quirúrgicos, lo que lleva al desarrollo de disfunción ventricular derecha por cambios en la geometría ventricular y predisposición a arritmias letales, con el consiguiente riesgo de reintervenciones. El implante valvular percutáneo pulmonar es una nueva alternativa terapéutica, menos invasiva en comparación con la quirúrgica, para pacientes seleccionados. Se realiza una revisión de las publicaciones médicas actuales disponibles y se describe la experiencia inicial del implante valvular pulmonar percutáneo en un centro colombiano de alta complejidad para el tratamiento de enfermedades cardiovasculares, en dos pacientes con disfunción del homoinjerto aórtico en posición pulmonar con doble lesión valvular, en los cuales el implante valvular pulmonar percutáneo fue una conducta exitosa. Se eligió a pacientes con cardiopatías congénitas, conductos quirúrgicos disfuncionales con estenosis o insuficiencia pulmonar significativa, y disfunción y dilatación ventricular derechas. Se empleó la técnica regular para el implante de la válvula pulmonar Melody, sin documentarse complicaciones durante el procedimiento ni al año de seguimiento. El implante percutáneo de la válvula pulmonar es un gran avance en el tratamiento de pacientes con cardiopatías congénitas, con resultados favorables a corto y mediano plazos, lo cual hace posible la restauración de la función ventricular con riesgo mínimo, frente al reemplazo quirúrgico en pacientes seleccionados.

Palabras clave: Estenosis de la válvula pulmonar. Insuficiencia de la válvula pulmonar. Prótesis e implantes. Procedimientos endovasculares. Cardiopatías congénitas.

\begin{abstract}
Patients with congenital heart disease that involves reconstruction of the right ventricular outflow tract must frequently undergo interventions derived from the limited useful life of the surgical canals, which leads to the development of right ventricular dysfunction due to changes in the ventricular geometry and predisposition to lethal arrhythmias, with the consequent risk of reinterventions. The percutaneous pulmonary valvular implant is a new therapeutic alternative, less invasive, compared to surgery, for selected patients. A review of the available literature is made and the initial experience of percutaneous pulmonary valve implantation in a Colombian center of high complexity for the treatment of cardiovascular diseases is described, in two
\end{abstract}

\section{Correspondencia:}

*Keerby Hernández

E-mail: keerby.cardioped@gmail.com
Fecha de recepción: 13-06-2019

Fecha de aceptación: 10-01-2020

DOI: 10.24875/ACM.20000252
Disponible en internet: 06-02-2020 Arch Cardiol Mex. 2020;90(3):313-320

www.archivoscardiologia.com

1405-9940/@ 2020 Instituto Nacional de Cardiología Ignacio Chávez. Publicado por Permanyer. Este es un artículo open access bajo la licencia CC BY-NC-ND (http://creativecommons.org/licenses/by-nc-nd/4.0/). 
patients with aortic homograft dysfunction in a pulmonary position with double valvular lesion, in which the percutaneous pulmonary valve implant was a successful strategy. Patients with congenital heart disease were chosen, with dysfunctional surgical conduits with stenosis or significant pulmonary insufficiency, with dysfunction and right ventricular dilatation. The standard technique for the implantation of the Melody pulmonary valve was used, without complications during the procedure or one year of follow-up. Percutaneous implantation of the pulmonary valve is a great advance in the management of patients with congenital heart diseases, with favorable results in the short and medium term, allowing the restoration of ventricular function with minimal risk, compared to surgical replacement in selected patients.

Key words: Pulmonary valve stenosis. Pulmonary valve insufficiency. Prostheses and implants. Endovascular procedures. Congenital heart disease.

\section{Introducción}

El tratamiento actual de las cardiopatías congénitas, junto con el avance de las técnicas de imagen no invasivas, el desarrollo de nuevos procedimientos quirúrgicos y el intervencionismo hemodinámico, ha contribuido al incremento de la sobrevida de los pacientes, pero también a la necesidad de mayores reintervenciones ${ }^{1}$. Por esa razón es importante aplicar nuevas técnicas que reduzcan la morbimortalidad y favorezcan un mayor tiempo sin intervenciones.

Las técnicas quirúrgicas que incluyen la continuidad entre el ventrículo derecho y la arteria pulmonar se acompañan a menudo de disfunción del tracto de salida del ventrículo derecho en el postoperatorio tardío Esta alteración fisiológica, sumada a la disfunción crónica de la válvula pulmonar, puede ocasionar efectos deletéreos en la función ventricular derecha, dilatación y predisposición a arritmias potencialmente letales, con un efecto negativo en la calidad de vida, la progresión sintomática y la disminución de la capacidad de ejercicio $^{3}$. La intervención temprana con el objetivo de restituir la funcionalidad valvular pulmonar puede prevenir e incluso revertir este proceso.

El implante percutáneo de la válvula pulmonar es una alternativa menos invasiva que la quirúrgica para el reemplazo valvular, que puede usarse en pacientes seleccionados $^{4}$. Desde su descripción en el año 2000 hasta la fecha, diferentes centros mundiales incorporaron esta práctica con más de 10,000 válvulas percutáneas implantadas en todo el mundo 5 . Los resultados a corto y mediano plazos muestran una tasa elevada de procedimientos exitosos con adecuada función valvular pulmonar $^{6}$ y bajas tasas de reintervención con mejoría de la clase funcional (NYHA) y los parámetros hemodinámi$\cos ^{7,8}$. Las causas principales de disfunción se relacionan con estenosis por constricción elástica temprana (recoil) y pérdida de la fuerza radial por fractura del stent ${ }^{9,10}$.

Numerosos estudios muestran que la resolución de la disfunción del tracto de salida del ventrículo derecho, a través del implante percutáneo valvular pulmonar, corrige los procesos de remodelación del ventrículo derecho y el grado de insuficiencia tricuspídea, además de mejorar la función biventricular ${ }^{6,11,12}$.

El objetivo de esta descripción de casos clínicos es mostrar la experiencia, en un centro colombiano para el tratamiento de enfermedades cardiovasculares, del implante valvular pulmonar percutáneo en dos pacientes con cardiopatía congénita corregida de manera inicial con homoinjerto aórtico en posición pulmonar y que desarrollaron con posterioridad doble lesión valvular pulmonar (estenosis e insuficiencia).

\section{Caso 1}

Paciente masculino de 16 años de edad, de $50 \mathrm{~kg}$ de peso, con antecedente de doble tracto de salida del ventrículo derecho, estenosis pulmonar, situs inversus abdominoauricular y dextrocardia, a quien se le realizó al principio una operación de Kawashima-Rastelli a los tres años de edad con un injerto valvular aórtico en posición pulmonar de $19 \mathrm{~mm}$ de diámetro. Durante el seguimiento ambulatorio se identificó deterioro progresivo de la clase funcional NYHA II, con desarrollo de disfunción del injerto, a los 13 años del implante, y doble lesión: estenosis pulmonar progresiva e insuficiencia pulmonar moderada. Durante el último control se registró un ecocardiograma que reveló un homoinjerto con estenosis grave, gradiente máximo de $77 \mathrm{mmHg}$ y medio de $47 \mathrm{mmHg}$ e insuficiencia moderada. Además, se observó una dilatación leve e hipertrofia ventricular derecha. Con estos hallazgos se solicitó resonancia magnética cardíaca. Se evidenció un ventrículo derecho dilatado con aneurisma del tracto de salida y un diámetro diastólico de $55 \mathrm{~mm}$, fracción regurgitante de $27 \%$, volumen telediastólico de $152 \mathrm{~cm}^{3} / \mathrm{m}^{2}$, telesistólico de $49 \mathrm{~cm}^{3} / \mathrm{m}^{2}$ y fracción de expulsión del $63 \%$; un ventrículo izquierdo dilatado, con un volumen telediastólico de $84 \mathrm{~cm}^{3} / \mathrm{m}^{2}$ y una fracción de expulsión del $60 \% \sin$ alteraciones segmentarias en la contractilidad. 


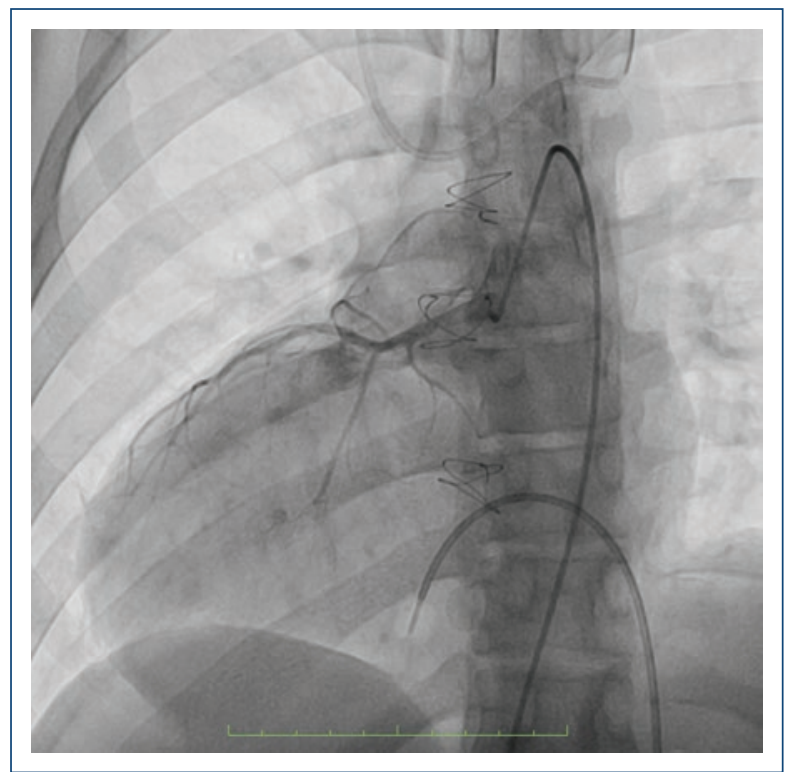

Figura 1. Imagen fluoroscópica anterior al implante valvular pulmonar: se observan arterias coronarias con origen y trayecto normales, sin defectos anatómicos que representen riesgo de oclusión.

Tras considerar el deterioro de la clase funcional y los hallazgos en los estudios, el paciente resultó elegible para implante de válvula pulmonar por vía percutánea. El procedimiento (véase la descripción más adelante) de implante valvular percutáneo se realizó en el laboratorio de hemodinamia bajo anestesia general, intubación orotraqueal y cuidado de anestesia cardiovascular; se obtuvieron coronariografias selectivas anteriores al implante para evaluar de forma detallada la anatomía coronaria, como se muestra en la figura 1. No se presentaron complicaciones durante el procedimiento. A continuación ingresó a la unidad de cuidados intensivos pediátricos durante un día sin necesidad de apoyo hemodinámico o ventilatorio. El ecocardiograma al siguiente dia posterior al implante valvular señaló homoinjerto en posición pulmonar, stent en adecuada posición, válvula Melody permeable y buena movilidad con un gradiente máximo de $25 \mathrm{mmHg}$ y medio de $13 \mathrm{mmHg}$, sin insuficiencia valvular pulmonar. Recibió el alta al segundo día de hospitalización.

\section{Caso 2}

Paciente de 18 años de vida, de $50 \mathrm{~kg}$ de peso, con antecedente de cardiopatía congénita tipo tetralogía de Fallot, que requirió fístula de Blalock-Taussig en el período neonatal y luego operación de Rastelli. Se implantó homoinjerto en posición pulmonar de $20 \mathrm{~mm}$ de diámetro y se identificó estenosis del homoinjerto que requirió angioplastia pulmonar al año siguiente, con buenos resultados hemodinámicos. Se continuó seguimiento ambulatorio en el cual, durante su evolución, desarrolló una doble lesión de estenosis pulmonar moderada e insuficiencia libre, por lo cual se solicitaron estudios complementarios para evaluar las implicaciones hemodinámicas y definir un plan cardiovascular. El ecocardiograma identificó una estenosis moderada del homoinjerto en posición pulmonar con gradiente máximo de $85 \mathrm{mmHg}$ y medio de $56 \mathrm{mmHg}$, insuficiencia libre pulmonar y dilatación del ventrículo derecho. La resonancia cardíaca delineó un ventrículo derecho dilatado, con hipocinesia difusa, fracción regurgitante de $25 \%$, fracción de expulsión del 57\%, volumen telediastólico de $103 \mathrm{ml} / \mathrm{m}^{2}$ y volumen telesistólico de $35 \mathrm{ml} / \mathrm{m}^{2}$. El ventrículo izquierdo mostró un tamaño normal, sin hipertrofia ni alteraciones de la contractilidad, fracción de expulsión del $61 \%$, volumen telediastólico de $64 \mathrm{ml} / \mathrm{m}^{2}$ y volumen telesistólico $25 \mathrm{ml} / \mathrm{m}^{2}$, con fracción de regurgitación de $25 \%$.

Se analizó en una junta médico-quirúrgica y, con base en los hallazgos de imágenes cardíacas, se consideró al paciente apto para implante percutáneo de válvula Melody. Se realizó el procedimiento en las mismas condiciones que el caso anterior y no se documentaron complicaciones durante el procedimiento. El ecocardiograma intracardíaco no reveló fuga paravalvular ni insuficiencia pulmonar. Se lo trasladó a la unidad de cuidados intensivos, donde permaneció por un día para vigilancia hemodinámica; el paciente presentó evolución clínica favorable, sin trastornos en el ritmo cardíaco o episodios de hipoperfusión. Con posterioridad se lo envió a la sala de hospitalización y egresó al segundo día de estancia hospitalaria.

\section{Descripción de la técnica del procedimiento}

Se efectúa cateterismo cardíaco derecho e izquierdo, con acceso femoral percutáneo. Se utiliza un catéter wedge para medir las presiones en la circulación derecha y un catéter helicoidal para angiografías en el VD. Se realiza intercambio por una guía Lunderquist de $0.35 \times 260 \mathrm{~cm}$ y sobre ésta se avanza un balón Atlas, el cual se expande en el tracto de salida del VD con coronariografía selectiva simultánea para descartar una compresión extrínseca (Fig. 2). El tracto de salida y el homoinjerto se preparan con implantación de un stent 


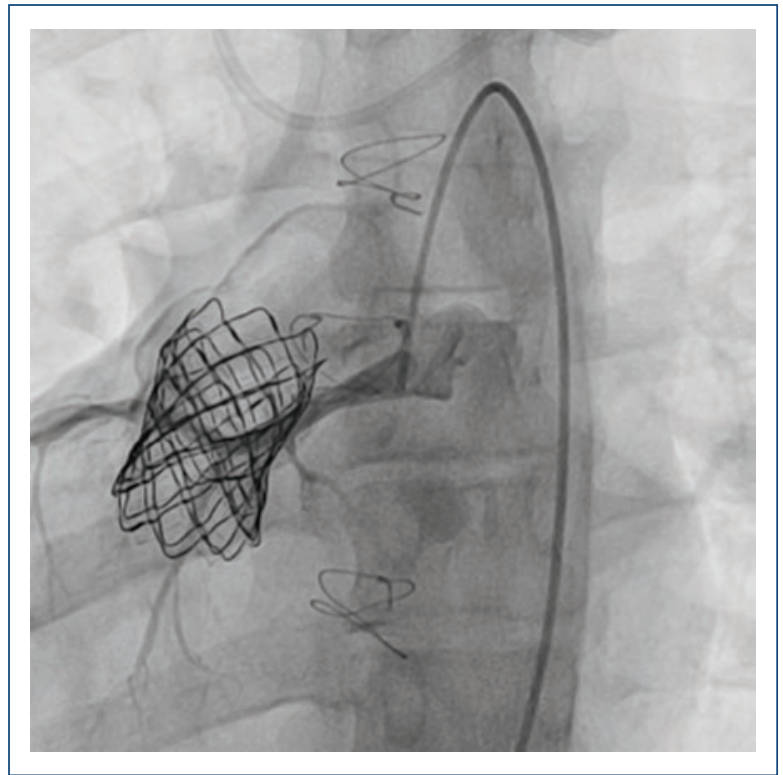

Figura 2. Imagen fluoroscópica realizada después del implante percutáneo valvular. No se demostró compresión extrínseca.

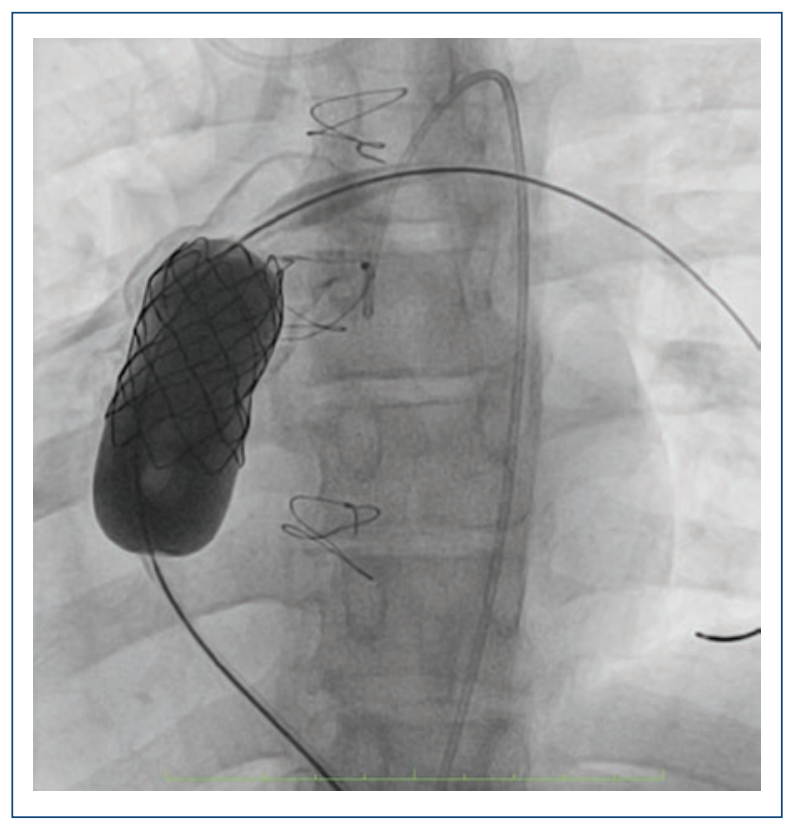

Figura 3. Angiografía del tracto de salida del ventrículo derecho. Implante de un stent cubierto CP sobre un balón Numed BIB (balloon in balloon) en el tracto de salida del ventrículo derecho sobre el homoinjerto aórtico en posición pulmonar.

cubierto CP sobre un balón Numed BIB (balloon in balloon), como se muestra en la figura 3 . Luego se prepara la válvula Melody (en uno de los casos tras

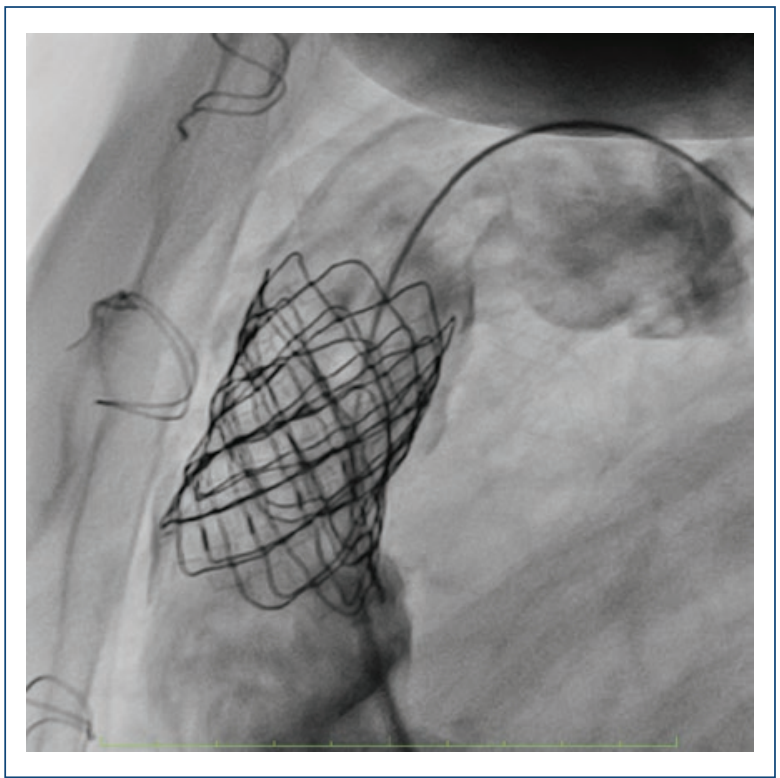

Figura 4. Imagen de válvula Melody implantada. Angiografía del conducto del tracto de salida del ventrículo derecho. Verificación de una posición adecuada de la válvula implantada.

acoplar un stent Andra XL adicional sobre esta prótesis) y se avanza con el sistema ENSAMBLE hasta la landing zone creada con el CP stent cubierto. Se infla el balón interno, se verifica una posición adecuada (Fig. 4) y luego se infla el externo para incidir de manera satisfactoria en la válvula. Se obtienen angiografías de control en la arteria pulmonar y el ventrículo derecho (Fig. 4) y se repiten las coronariografías de control para descartar compresión. Los principales resultados obtenidos en el laboratorio de hemodinamia se presentan en la tabla 1 y para cada uno de los pacientes notificados.

El seguimiento ambulatorio de los dos pacientes se realizó al mes del procedimiento con ecocardiograma y electrocardiograma, y luego a los seis meses y al año tras el procedimiento. Ninguno requirió reintervención. No se presentaron alteraciones en el ritmo cardíaco. Uno de los pacientes con clase funcional NYHA II en el momento del implante refirió mejoría en la tolerancia al ejercicio un mes después de la intervención. Los dos pacientes recibieron tratamiento antiagregante plaquetario con ácido acetilsalicílico durante los primeros seis meses posteriores al procedimiento. La evaluación ecocardiográfica ambulatoria mostró una mejoría significativa de los volúmenes pulmonares y la evaluación de la funcionalidad del ventrículo derecho medida por TAPSE. No se observó insuficiencia pulmonar residual ni fractura de la 
Tabla 1. Valores hemodinámicos

\begin{tabular}{|l|c|c|c|c|}
\hline \multicolumn{5}{|c|}{ Estudio hemodinámico, caso 1 } \\
\hline Cavidad & PS* & PD* & PD2* & PM* $^{*}$ \\
\hline VD* $^{*}$ & 54 & & 10 & \\
\hline Ao. desc.* & 81 & 46 & & 52 \\
\hline \multicolumn{4}{|c|}{ Postimplantación de válvula Melody 22} \\
\hline AP* & 34 & 16 & & 23 \\
\hline VD* & 35 & 0 & 10 & \\
\hline Ao. desc.* & 100 & 65 & & 61 \\
\hline Tiempo de cateterismo, 130 minutos \\
\hline Tiempo de fluoroscopia: $23-26$ minutos
\end{tabular}

\begin{tabular}{|c|c|c|c|c|}
\hline \multicolumn{5}{|c|}{ Estudio hemodinámico, caso 2} \\
\hline$A D^{*}$ & 10 & & & \\
\hline$V D^{*}$ & 88 & 0 & 13 & \\
\hline$A P^{*}$ & 25 & 10 & & 15 \\
\hline RDAP* & 25 & 8 & & 16 \\
\hline RIAP* & 20 & 10 & & 15 \\
\hline Cuña izquierda & & & & 8 \\
\hline Cuña derecha & & & & 10 \\
\hline TSVD* & 85 & & & \\
\hline $\mathrm{VI}^{*}$ & 90 & 0 & 8 & \\
\hline Ao. asc.* & 95 & 62 & & 77 \\
\hline Ao. desc.. ${ }^{*}$ & 90 & 60 & & 78 \\
\hline \multicolumn{5}{|c|}{ Postimplantación de válvula Melody 22} \\
\hline VCS* & & & & 8 \\
\hline$A D^{*}$ & & & & 8 \\
\hline$V D^{*}$ & 48 & 0 & 10 & \\
\hline$A P^{*}$ & 23 & 8 & & 17 \\
\hline RDAP* & 22 & 6 & & 17 \\
\hline Cuña & & & & 8 \\
\hline Intrastent & 22 & 10 & 14 & \\
\hline Aorta & 110 & 70 & & 84 \\
\hline \multicolumn{5}{|c|}{ Tiempo de cateterismo: 160 minutos } \\
\hline Tiempo de fluorc & $68 \mathrm{r}$ & & & \\
\hline
\end{tabular}

VD: ventrículo derecho; Ao. desc.: aorta descendente; AP: arteria pulmonar; PS: presión sistólica; PD1: presión diastólica; PD2: presión de final de la diástole PM: presión media; VCS: vena cava superior; AD: aurícula derecha; RDAP: rama derecha de la arteria pulmonar; RIAP: rama izquierda de la arteria pulmonar; TSVD: tracto de salida del ventrículo derecho; VI: ventrículo izquierdo; Ao. asc.: aorta ascendente.

válvula Melody. No se registraron complicaciones tardías relacionadas con el implante percutáneo al año de seguimiento.

\section{Discusión}

Los procedimientos quirúrgicos para las cardiopatías congénitas que incluyen el tracto de salida del ventrículo derecho y la arteria pulmonar implican muchas veces la implantación de un conducto para restituir la conexión entre el ventrículo derecho y la arteria pulmonar. En pacientes pediátricos, estos conductos deben reemplazarse con frecuencia y muestran una vida útil limitada que varía según sean la edad del paciente y el material utilizado $^{13}$ debido al crecimiento somático y la degeneración del conducto que puede ocasionar estenosis o insuficiencia. Esto representa una remodelación ventricular con subsiguiente dilatación y consecuencias deletéreas para la funcionalidad ventricular derecha ${ }^{14}$ y ello eleva la morbilidad inherente y la necesidad de reintervenciones quirúrgicas en períodos menores de 10 años $^{15}$.

Los avances tecnológicos en el tratamiento intervencionista percutáneo han revolucionado la atención de los pacientes con cardiopatías congénitas. El implante percutáneo valvular pulmonar se describió de forma original en el año $2000^{16}$ y lo aprobó la Food and Drug Administration (FDA) como un dispositivo de uso humano en enero de 2010 para tratar conductos disfuncionales en el tracto de salida del ventrículo derecho ${ }^{7}$. Es una alternativa novedosa y menos invasiva que el procedimiento quirúrgico de recambio valvular pulmonar.

Hoy en día existen dos dispositivos aprobados para el implante de válvula pulmonar percutánea: la válvula Melody (Medtronic) es un dispositivo diseñado de tejido yugular bovino, con una válvula central de valvas naturales que se abren y coaptan bajo condiciones óptimas de presión y está suturada sobre un stent de platino-iridio que se encuentra sobre un $C P$ stent con guía fluoroscópica y un sistema de liberación "balón en balón" (BIB) Ensemble ${ }^{T M}$, que cuenta con una funda integrada que protege a la válvula durante la liberación controlada. El sistema se encuentra disponible en el comercio con diámetro externo de 18,20 y $22 \mathrm{~mm}^{17}$. La válvula Edwards SAPIEN XT es una válvula elaborada con tejido pericárdico bovino en un stent de acero inoxidable de 14 a $16 \mathrm{~mm}$, con un dispositivo de sellado que cubre la parte proximal del stent con el objetivo de evitar que se presenten fugas paravalvulares, e incluye al sistema de liberación Nova Flex ${ }^{\mathrm{TM}}$ que estabiliza el cuerpo del balón durante la implantación. Se encuentra disponible en 23, 26 y 29 mm de diámetro. Esta válvula utiliza el sistema de liberación que requiere guías hidrofílicas 22-24 Fr y los datos sobre la funcionalidad de la válvula SAPIEN XT son limitados en la actualidad por un número bajo de pacientes ${ }^{18,19}$. 
La evidencia en el implante valvular percutáneo con válvula Melody proviene de estudios analíticos y descriptivos multicéntricos, con gran experiencia sobre todo en EE.UU. y Europa, con resultados excelentes a corto y mediano plazos ${ }^{8,20}$. En Latinoamérica se ha documentado la práctica en centros de referencia de cardiología intervencionista en Brasil y Argentina, y se ha observado que la técnica es factible, segura y eficaz ${ }^{2}$.

Este informe de casos describe por primera vez la experiencia del implante valvular pulmonar percutáneo en un centro colombiano de alta complejidad para el tratamiento de enfermedades cardiovasculares, y cuenta con un equipo multidisciplinario capacitado en el tratamiento intervencionista de las cardiopatías congénitas, con resultados exitosos a corto plazo.

Según las recomendaciones actuales de la American Heart Association (AHA), se recomienda el reemplazo percutáneo de la válvula pulmonar en pacientes con conductos quirúrgicos de ventrículo derecho a arteria pulmonar, con regurgitación moderada a grave o estenosis, que producen sobrecarga de presión o volumen al ventrículo derecho, siempre que el paciente cumpla con los criterios de inclusión o exclusión para la válvula disponible ${ }^{21}$. En el caso específico de la válvula Melody se requiere además un diámetro circunferencial del homoinjerto de 16 a $22 \mathrm{~mm}$. Los dos casos notificados satisfacen estos requisitos: son portadores de homoinjerto aórtico en posición pulmonar de 19 y $20 \mathrm{~mm}$ de diámetro, respectivamente, tienen peso superior a $30 \mathrm{~kg}$, mostraron doble lesión de la válvula moderada a grave y fracción de regurgitación mayor de $25 \%$ con dilatación e hipertrofia ventricular derecha y relaciones entre los volúmenes de final de la diástole (VD/VI) mayores de 1.7. De manera adicional se identificó en el seguimiento deterioro progresivo de la clase funcional NYHA en uno de los casos.

El procedimiento se practicó en el laboratorio de hemodinamia, con oxígeno al 100\% bajo intubación orotraqueal, con cuidado de anestesiología cardiovascular, previo consentimiento informado. Las vías de acceso fueron la venosa femoral y la arterial para angiografía aórtica y coronariografía selectivas, con el fin de detectar alguna anatomía coronaria susceptible de compresión. Se administraron heparina intravenosa y profilaxis antibiótica. El conducto se dilató previamente con balones de diámetros progresivos y se realizaron coronariografías selectivas durante la prueba de balón en el tracto de salida del ventrículo derecho en ambos casos, sin registrarse cambios hemodinámicos, angiográficos o electrocardiográficos que sugirieran defectos de perfusión. La válvula implantada percutánea en los dos casos fue una Melody de $22 \mathrm{~mm}$, sobre el conducto quirúrgico, liberada por el sistema Ensemble diseñado para este fin. Se siguieron de modo riguroso las recomendaciones para el implante. Los datos hemodinámicos inmediatos posteriores al implante de la válvula Melody se encuentran en la tablas 1 para cada paciente, respectivamente.

Las complicaciones agudas perioperatorias se han descrito en las publicaciones médicas en un $6 \%$ con un $2.7 \%$ de complicaciones que ponen en peligro la vida del paciente o requieren intervención inmediata, como ruptura del conducto, perforación y obstrucción de arterias pulmonares, lesiones o desplazamiento valvular y compresión de arterias coronarias debido a la intervención del tracto de salida del VD con la localización del stent $t^{7,20}$. Esta última se acompaña de alta mortalidad temprana y de ahí la importancia de conocer la anatomía coronaria, que permita realizar una planificación anterior al implante valvular y evitar desenlaces desfavorables. En los dos casos expuestos se realizó coronariografía selectivas antes y después del implante valvular percutáneo para evaluar compresión extrínseca y no se documentaron complicaciones durante el procedimiento.

La intervención se consideró exitosa en los dos casos y la válvula Melody implantada tuvo una localización adecuada, con disminución del gradiente pico del ventrículo derecho a arteria pulmonar y de la relación de las presiones entre el ventrículo derecho y la aorta, con insuficiencia pulmonar residual leve y gradiente encontrado entre el ventrículo derecho y la arteria pulmonar menor de $35 \mathrm{mmHg}$. En ninguno de los dos casos fue necesario el explante en las siguientes 24 horas del procedimiento?.

En el seguimiento a corto plazo se ha relacionado el desarrollo de endocarditis bacteriana como primera causa de explante quirúrgico y mortalidad secundaria a sepsis; la tasa anual informada es del $2.4 \%$ paciente-año ${ }^{18}$. La mayoría de los casos revela una adecuada respuesta al tratamiento antimicrobiano, incluidos mecanismos fisiopatológicos como alteraciones en el patrón de flujo por el gradiente residual, trombosis in situ e inadecuada administración de profilaxis antibiótica durante el procedimiento, así como mayor adhesión bacteriana en la pared venosa yugular bovina para algunas sepas de $S$. aureus ${ }^{22}$. En la institución de los autores se adoptó el protocolo de antibioticoterapia profiláctica durante los procedimientos de intervención hemodinámica con cefalosporinas de primera generación, además de la implementación de adecuadas técnicas de asepsia y antisepsia. No se documentaron endocarditis en ninguno de los casos. 
Los trastornos del ritmo cardíaco también se han notificado en las publicaciones médicas, en particular aparición de novo tras el implante, sobre todo extrasístoles ventriculares o taquicardia ventricular no sosteni$\mathrm{da}$, de carácter transitorio y autolimitadas en la mayor parte de los $\operatorname{casos}^{23}$. En la experiencia de los autores no se presentaron alteraciones en el ritmo cardíaco.

El procedimiento ha presentado resultados promisorios en los diferentes estudios, con tasas elevadas de éxito ${ }^{24}$. La sobrevida se ha determinado en $98 \%$ a cinco años, con ausencia de reintervención en $98 \pm 1 \%$ a los tres años y de $91 \pm 3 \%$ a los cinco años, con períodos libres más cortos en pacientes con mayores gradientes de obstrucción en el tracto de salida del ventrículo derecho ${ }^{7}$. La tasa de ausencia de disfunción de la válvula implantada transcatéter en un registro de seguimiento a un año se informó en $96.9 \%$ y en $86.4 \%$ a los 27 me$\operatorname{ses}^{10}$. Las causas principales de disfunción se han relacionado con estenosis por el recoil y fractura del stent por pérdida de la fuerza radial9,10. Los factores relacionados con ruptura son pacientes con tractos de salida del ventrículo derecho muy obstruidos, compresión externa por la pared torácica cuando la válvula implantada se localiza directamente detrás de la pared, menor edad en el momento de la intervención y menor diámetro angiográficos del conducto; por el contrario, se ha descrito como factor protector un sitio de implante protegido por una bioprótesis valvular o un stent previo ${ }^{10,25}$, este último el caso de los dos pacientes expuestos.

Este informe sustenta la noción de que el reemplazo valvular pulmonar percutáneo es un procedimiento de intervención útil en el tratamiento de la obstrucción del tracto de salida del ventrículo derecho y regurgitación pulmonar postoperatoria, relacionados con efectos deletéreos como remodelación y disfunción ventricular derecha, así como insuficiencia valvular tricúspide progresiva. Al restaurar la función valvular pulmonar con implante percutáneo, mejora la tolerancia al ejercicio, se reducen los volúmenes del ventrículo derecho y mejora la funcionalidad biventricular al mejorar el llenado del ventrículo izquierdo ${ }^{26}$, además de producir reducciones clínicamente relevantes en la insuficiencia tricúspide que persisten aun después de cinco años de seguimiento ${ }^{12}$.

\section{Conclusión}

El reemplazo valvular pulmonar percutáneo se considera una alternativa atractiva a la sustitución valvular quirúrgica para restaurar la función pulmonar en pacientes con disfunción del tracto de salida del ventrículo derecho; es seguro, eficaz y menos invasivo, con tasas actuales de éxito y resultados optimistas en el seguimiento a corto y mediano plazos en pacientes seleccionados, que pueden además adoptar los cardiólogos pediatras intervencionistas capacitados.

La incidencia notificada en las publicaciones médicas de episodios adversos sirve como base para disminuir y tratar si es posible los factores de riesgo relacionados con desenlaces inadecuados. Se necesita un seguimiento prolongado para evaluar la incidencia de complicaciones tardías.

\section{Agradecimientos}

Los autores agradecen de manera muy especial al Dr. J. L. Zunzunegui, quien compartió su experiencia y conocimiento con este centro e hizo posible que estos dos primeros casos de implante valvular percutáneo pulmonar se llevaran a cabo.

\section{Financiamiento}

Esta investigación no ha recibido ninguna beca específica de agencias de los sectores público, comercial o sin ánimo de lucro.

\section{Conflicto de intereses}

Los autores declaran que no tienen ningún conflicto de intereses.

\section{Responsabilidades éticas}

Protección de personas y animales. Los autores declaran que para esta investigación no se han realiza experimentos en seres humanos ni en animales.

Confidencialidad de los datos. Los autores declaran que han seguido los protocolos de su centro de trabajo sobre la publicación de datos de pacientes.

Derecho a la privacidad y consentimiento informado. Los autores han obtenido el consentimiento informado de los pacientes y/o sujetos referidos en el artículo. Este documento obra en poder del autor de correspondencia.

\section{Bibliografía}

1. Solana-Gracia R, Rueda F, Betrián $P$, Gutiérrez-Larraya F, Del Cerro MJ, Pan $\mathrm{M}$, et al. Pediatrics spanish registry of percutaneous melody pulmonary valve implantation in patients younger than 18 years. Rev Esp Cardiol (Engl Ed). 2018;71(4):283-90.

2. Ribeiro MS, Pedra CAC, Costa RN, Rossi RI, Manica JoL, Nascimento WTM, et al. Experiência inicial com o implante percutâneo da válvula Melody ${ }^{\circledR}$ no Brasil. Revista Brasileira de Cardiologia Invasiva. 2014;22:275-85 
3. Shimazaki Y, Blackstone EH, Kirklin JW. The natural history of isolated congenital pulmonary valve incompetence: surgical implications. Thorac Cardiovasc Surg. 1984;32(4):257-9.

4. Rosengart TK, Feldman T, Borger MA, Vassiliades TA, Gillinov AM, Hoercher $\mathrm{KJ}$, et al. Percutaneous and minimally invasive valve procedures: a scientific statement from the American Heart Association Council on Cardiovascular Surgery and Anesthesia, Council on Clinical Cardiology, Functional Genomics and Translational Biology Interdisciplinary Working Group, and Quality of Care and Outcomes Research Interdisciplinary Working Group. Circulation. 2008;117(13):1750-67.

5. Gillespie MJ, Rome JJ, Levi DS, Williams RJ, Rhodes JF, Cheatham JP, et al. Melody valve implant within failed bioprosthetic valves in the pulmonary position: a multicenter experience. Circ Cardiovasc Interv. 2012;5(6):862-70.

6. McElhinney DB, Hellenbrand WE, Zahn EM, Jones TK, Cheatham JP Lock JE, et al. Short- and medium-term outcomes after transcatheter pulmonary valve placement in the expanded multicenter US melody valve trial. Circulation. 2010:122(5):507-16.

7. Armstrong AK, Balzer DT, Cabalka AK, Gray RG, Javois AJ, Moore JW et al. One-year follow-up of the Melody transcatheter pulmonary valve multicenter post-approval study. JACC Cardiovasc Interv. 2014 $7(11): 1254-62$.

8. Cheatham JP, Hellenbrand WE, Zahn EM, Jones TK, Berman DP Vincent JA, et al. Clinical and hemodynamic outcomes up to 7 years after transcatheter pulmonary valve replacement in the US melody valve investigational device exemption trial. Circulation. 2015;131(22):1960-70.

9. Torres AJ. Pre-stenting and Melody valve stent fracture: When more is less. JACC Cardiovasc Interv. 2017;10(17):1760-2

10. McElhinney DB, Cheatham JP, Jones TK, Lock JE, Vincent JA, Zahn EM et al. Stent fracture, valve dysfunction, and right ventricular outflow tract reintervention after transcatheter pulmonary valve implantation: patient-related and procedural risk factors in the US Melody Valve Trial. Circ Cardiovasc Interv. 2011;4(6):602-14.

11. Frigiola A, Tsang V, Bull C, Coats L, Khambadkone S, Derrick G, et al Biventricular response after pulmonary valve replacement for right ventricular outflow tract dysfunction: is age a predictor of outcome? Circulation. 2008;118(14 Suppl):S182-90.

12. Jones TK, Rome JJ, Armstrong AK, Berger F, Hellenbrand WE Cabalka AK, et al. Transcatheter pulmonary valve replacement reduces tricuspid regurgitation in patients with right ventricular volume/pressure overload. J Am Coll Cardiol. 2016;68(14):1525-35.

13. Homann M, Haehnel JC, Mendler N, Paek SU, Holper K, Meisner H, et al. Reconstruction of the RVOT with valved biological conduits: 25 years experience with allografts and xenografts. Eur J Cardiothorac Surg 2000;17(6):624-30.
14. Eicken A, Ewert $P$, Hager A, Peters B, Fratz $S$, Kuehne $T$, et al Percutaneous pulmonary valve implantation: two-centre experience with more than 100 patients. Eur Heart J. 2011;32(10):1260-5.

15. Askovich B, Hawkins JA, Sower CT, Minich LL, Tani LY, Stoddard G, et al. Right ventricle-to-pulmonary artery conduit longevity: is it related to allograft size? Ann Thorac Surg. 2007;84(3):907-11; discussion 11-2.

16. Ansari MM, Cardoso R, Garcia D, Sandhu S, Horlick E, Brinster D, et al. Percutaneous pulmonary valve implantation: present status and evolving future. J Am Coll Cardiol. 2015;66(20):2246-55.

17. Wagner R, Daehnert I, Lurz P. Percutaneous pulmonary and tricuspid valve implantations: an update. World J Cardiol. 2015;7(4):167-77.

18. Boone RH, Webb JG, Horlick E, Benson L, Cao QL, Nadeem N, et al. Transcatheter pulmonary valve implantation using the Edwards SAPIEN transcatheter heart valve. Catheter Cardiovasc Interv. 2010;75(2):286-94

19. Haas NA, Moysich A, Neudorf U, Mortezaeian H, Abdel-Wahab M, Schneider $\mathrm{H}$, et al. Percutaneous implantation of the Edwards SAPIEN(TM) pulmonic valve: initial results in the first 22 patients. Clin Res Cardiol. 2013;102(2):119-28.

20. Bergersen L, Benson LN, Gillespie MJ, Cheatham SL, Crean AM, Hor KN, et al. Harmony Feasibility Trial: acute and short-term outcomes with a self-expanding transcatheter pulmonary valve. JACC CardiovasC Interv. 2017;10(17):1763-73.

21. Feltes TF, Bacha E, Beekman RH, Cheatham JP, Feinstein JA, Gomes AS, et al. Indications for cardiac catheterization and intervention in pediatric cardiac disease: a scientific statement from the American Heart Association. Circulation. 2011;123(22):2607-52.

22. Jalal Z, Galmiche L, Lebeaux D, Villemain O, Brugada G, Patel M, et al. Selective propensity of bovine jugular vein material to bacterial adhesions: an in-vitro study. Int J Cardiol. 2015;198:201-5.

23. Nguyen HH, Shahanavaz S, Van Hare GF, Balzer DT, Nicolas R, Avar Silva JN. Percutaneous pulmonary valve implantation alters electrophysiologic substrate. J Am Heart Assoc. 2016;5(10).

24. Lurz P, Coats L, Khambadkone S, Nordmeyer J, Boudjemline Y, Schievano $S$, et al. Percutaneous pulmonary valve implantation: impact of evolving technology and learning curve on clinical outcome. Circulation. 2008;117(15):1964-72

25. Nordmeyer J, Khambadkone S, Coats L, Schievano S, Lurz P, Parenzan $\mathrm{G}$, et al. Risk stratification, systematic classification, and anticipatory management strategies for stent fracture after percutaneous pulmonary valve implantation. Circulation. 2007;115(11):1392-7.

26. Secchi F, Resta EC, Cannaò PM, Tresoldi S, Butera G, Carminati M, et al. Four-year cardiac magnetic resonance (CMR) follow-up of patients treated with percutaneous pulmonary valve stent implantation. Eur Radiol. 2015;25(12):3606-13. 